\title{
A Recursive Frequency Estimator Using Linear Prediction and a Kalman-Filter-Based Iterative Algorithm
}

\author{
Z. G. Zhang, S. C. Chan, Member, IEEE, and K. M. Tsui
}

\begin{abstract}
This paper proposes a new Kalman-filter-based recursive frequency estimator for discrete-time multicomponent sinusoidal signals whose frequencies may be time-varying. The frequency estimator is based on the linear prediction approach and it employs the Kalman filter to track the linear prediction coefficients (LPCs) recursively. Frequencies of the sinusoids can then be computed using the estimated LPCs. Due to the coloredness of the linear prediction error, an iterative algorithm is employed to estimate the covariance matrix of the prediction error and the LPCs alternately in the Kalman filter in order to improve the tracking performance. Simulation results show that the proposed Kalman-filter-based iterative frequency estimator can achieve better tracking results than the conventional recursive least-squares-based estimators.
\end{abstract}

Index Terms-Iterative method, Kalman filter, linear prediction, recursive frequency estimation.

\section{INTRODUCTION}

$\mathbf{T}$ HE frequency estimation of real-valued sinusoidal signals has found important applications in speech processing [1], biomedical engineering [2], and many other areas. The problem is to estimate the frequencies of a sinusoidal signal from its discrete-time noisy measurements

$$
y(n)=s(n)+q(n), \quad n=0,1, \ldots, N-1
$$

where $s(n)=\sum_{m=1}^{M} \alpha_{m} \cos \left(2 \pi w_{m} n+\phi_{m}\right)$ are the discretetime samples of the noise-free real-valued sinusoidal signal, where $\alpha_{m}>0, ; w_{m} \in(0,0.5)$, and $\phi_{m} \in[0,2 \pi)$ are the amplitude, frequency, and phase of the $m$ th frequency component, respectively. $q(n)$ is the additive white Gaussian noise (AWGN) with zero mean and variance $\sigma_{q}^{2}$. For simplicity, we assume that the number of sinusoids $M$ is known and the frequencies are distinct, i.e., $w_{m_{1}} \neq w_{m_{2}}$ when $m_{1} \neq m_{2}$.

A number of methods were proposed to solve the above frequency estimation problem for the discrete-time data samples. These include the Yule-Walker method [3], the maximum-likelihood method [3], subspace-based approaches such as the MUSIC algorithm and the ESPRIT algorithm [3], and the classical linear prediction (LP) approach [4]. In the automatic control community, an adaptive notch filter-based method was

Manuscript received May 23, 2007; revised November 15, 2007. This paper was recommended by Associate Editor A. Vicino.

The authors are with the Department of Electrical and Electronic Engineering, The University of Hong Kong, Hong Kong (e-mail: zgzhang@eee.hku.hk; scchan@eee.hku.hk; kmtsui@eee.hku.hk).

Digital Object Identifier 10.1109/TCSII.2007.916837 also studied extensively for online frequency estimation of continuous-time signals [5], [6].

In [7], it was shown that the prediction residuals of the LP approach are correlated and colored so that a weighted leastsquares (WLS) frequency estimator was proposed to whiten the residuals. The frequency components and the weighting matrix were estimated alternately until convergence and an iterative WLS algorithm was proposed. The algorithm in [7] was intended for batch processing of a block of observations, and it works well for stationary signals with fixed frequencies. However, when the frequencies of a sinusoidal signal change considerably with time, the performance of the batch-processing algorithm will degrade substantially.

Recently, a new time-recursive QR-decomposition (QRD)based frequency estimator using the LP approach in [7] was proposed in [8]. It reduces the arithmetic complexity for online applications and improves the numerical property of the algorithm in finite word-length implementation. This QRD-based weighted recursive least-squares (WRLS) algorithm was shown to work well for tracking of static and slowly varying frequency components.

In this paper, we propose a new frequency estimator and extend the approach in [8] by incorporating state dynamics into the linear prediction coefficients (LPCs) based on the Kalman filter. The advantage of the LP approach is that the prediction residuals are linear function of the LPCs. Therefore, the conventional Kalman filter with appropriately chosen noise parameters can be employed to estimate the LPCs. The Kalman filter is a generalization of the WRLS algorithm and it allows prior information of the state dynamics to be incorporated into the estimation process. The Kalman filter has been proved to be an optimal estimator in the minimum mean-square-error (MMSE) sense when the signal and noise are jointly Gaussian [9], [10].

In the proposed Kalman-filter-based frequency estimation algorithm, the LPCs are assumed to be the system state, which can be described by some models such as an autoregressive (AR) process. A linear state-space model can then be established, and the Kalman filter is employed to track the LPCs and hence the frequencies recursively. Since the LP residuals are colored, the covariance matrix of the LP residuals, which are the measurement noise in the Kalman filter framework, has to be updated recursively. Therefore, an iterative algorithm based on the Kalman filter is developed to estimate the LPCs and measurement noise covariance matrix iteratively so as to further improve the tracking performance. Simulation results show that the 
proposed algorithm gives better tracking capabilities than the WRLS method in nonstationary environment because the latter is more sensitive to the changes of noise variance [11], [12].

This paper is organized as follows. In Section II, the LP approach and the WLS algorithm for frequency estimation are revisited. The Kalman-filter-based frequency estimator for time-varying frequency components is introduced in Section III. Section IV studied the noise covariance estimation in the Kalman-filter-based frequency estimator and proposed an improved iterative Kalman-filter-based frequency estimator. Simulation results and comparison are presented in Section V. Finally, conclusions are drawn in Section VI.

\section{LINEAR PREDICTION APPROACH}

It was observed by Prony [3] that the current sample of $s(n)$ in (1) can be expressed as a linear combination of its $2 M$ past samples based on the idea of LP, that is

$$
s(n)=\sum_{i=1}^{2 M} b_{i} s(n-i)
$$

where $b_{i}, i=1, \ldots, 2 M$, are called the LPCs. By the symmetric property of the LPCs of real sinusoids, $b_{i}=b_{2 M-i}$ and $b_{0}=b_{2 M}=-1$, (2) can be rewritten as

$$
\begin{aligned}
& s(n)+s(n-2 M) \\
& =\sum_{i=1}^{M-1} b_{i}[s(n-i)+s(n-2 M+i)]+b_{M} s(n-M) .
\end{aligned}
$$

Using the observations from (1) and $s(n)=y(n)-q(n)$, an overdetermined system can then be set up as follows:

$$
\mathrm{Z}=\mathbf{Y b}+\mathbf{e}
$$

where $\mathbf{b}=\left[b_{1}, \ldots, b_{M}\right]^{T}$ and $\mathbf{Y}$ and $\mathbf{Z}$ are defined as shown at the bottom of the page. $\mathbf{e}=[e(N-1), e(N-2), \ldots, e(2 M)]^{T}$ is the prediction residual vector with entries

$$
\begin{aligned}
e(n)= & y(n)+y(n-2 M) \\
& -\sum_{i=1}^{M-1} b_{i}[y(n-i)+y(n-2 M+i)]-b_{M} y(n-M) \\
= & -\sum_{i=0}^{M-1} b_{i}[q(n-i)+q(n-2 M+i)]-b_{M} q(n-M) \\
& \text { for } n=N-1, \ldots, 2 M .
\end{aligned}
$$

If $e(n)$ is a zero-mean independent identically distributed (i.i.d.) Gaussian random process, then the maximum-likelihood estimator (MLE) of $\mathbf{b}$ is identical to the least-squares (LS) estimator with the cost function

$$
J(\mathbf{b})=\sum_{n=2 M}^{N-1} e^{2}(n)=\mathbf{e}^{T} \cdot \mathbf{e}=(\mathbf{Z}-\mathbf{Y} \mathbf{b})^{T}(\mathbf{Z}-\mathbf{Y} \mathbf{b}) .
$$

However, it can be seen from (5) that $e(n)$ is obtained from linear combinations of $q(n)$ 's and hence it is correlated. A WLS algorithm should therefore be used to improve the estimation accuracy [7]. More precisely, let e be a zero mean Gaussian process with probability density function (p.d.f.) given by

$$
p(\mathbf{e})=\frac{1}{\left(2 \pi\left|\mathbf{R}_{e}\right|\right)^{(N-2 M) / 2}} \exp \left(-\left(\frac{1}{2}\right) \mathbf{e}^{T} \mathbf{R}_{e}^{-1} \mathbf{e}\right)
$$

where $\mathbf{R}_{e}=E\left[\mathbf{e e}^{H}\right]$ is the covariance matrix of e. The likelihood function is maximized when $\left((1 / 2) \mathbf{e}^{T} \mathbf{R}_{e}^{-1} \mathbf{e}\right)$ is minimized. Thus, we have the following WLS problem:

$$
\hat{\mathbf{b}}=\arg \min _{\hat{\mathbf{b}}}\left(\mathbf{e}^{T} \mathbf{R}_{e}^{-1} \mathbf{e}\right) .
$$

As a result, the LS cost function (6) should be modified to a WLS cost function as

$$
J(\hat{\mathbf{b}})=\mathbf{e}^{T} \mathbf{W e}=(\mathbf{Z}-\mathbf{Y} \hat{\mathbf{b}})^{T} \mathbf{W}(\mathbf{Z}-\mathbf{Y} \hat{\mathbf{b}})
$$

where $\mathbf{W}=\mathbf{R}_{e}^{-1}$ is the weighting matrix. The corresponding WLS solution is

$$
\hat{\mathbf{b}}=\left(\mathbf{Y}^{T} \mathbf{W} \mathbf{Y}\right)^{-1} \mathbf{Y}^{T} \mathbf{W Z} .
$$

Since $\mathbf{W}=\mathbf{R}_{e}^{-1}$ is unknown during the estimation of $\hat{\mathbf{b}}$, an iterative WLS algorithm is employed to estimate the parameters successively. More precisely, $\hat{\mathbf{b}}$ is estimated initially by the LP approach in (10) with $\mathbf{W}=\mathbf{I}$. Using the estimated $\hat{\mathbf{b}}$, we can construct $\mathbf{W}^{-1}$ using the algorithm mentioned in [7] and then take the inverse to obtain $\mathbf{W}$. $\hat{\mathbf{b}}$ is then updated again and the two processes are performed alternately until convergence.

After the LPC $\mathbf{b}=\left[\hat{b}_{1}, \ldots, \hat{b}_{M}\right]^{T}$ is estimated, the frequencies are computed by solving the polynomial equation [13]

$$
\sum_{i=0}^{2 M} \hat{b}_{i} \exp \left(-j 2 \pi w_{m} i\right)=0
$$

$$
\begin{aligned}
& \mathbf{Y}=\left[\begin{array}{ccccc}
y(N-2)+y(N-2 M) & y(N-3)+y(N-2 M+1) & & y(N-M)+y(N-M-2) & y(N-M-1) \\
\vdots & \vdots & \ddots & \vdots & \vdots \\
y(2 M-1)+y(1) & y(2 M-2)+y(2) & & y(M+1)+y(M-1) & y(M)
\end{array}\right] \\
& \mathbf{Z}=[y(N-1)+y(N-2 M-1), y(N-2)+y(N-2 M-2), \ldots, y(2 M+1)+y(1), y(2 M)+y(0)]^{T}
\end{aligned}
$$


where $j=\sqrt{-1}$ and $\hat{b}_{0}=-1$. $\left[\hat{b}_{M+1}, \ldots, \hat{b}_{2 M}\right]$ is obtained using the symmetric property of the LPCs: $\hat{b}_{i}=\hat{b}_{2 M-i}$. Note that $\hat{b}_{i}$ is extended to include the case $i=0$. This implies that the phases of the roots of (11) are the $2 M$ frequencies. For real sinusoids, only $M$ positive frequencies are needed.

\section{KALMAN-FILTER-BASED FREQUENCY ESTIMATOR}

The LP-based frequency estimation introduced in the previous section is a batch processing algorithm, where the frequencies are obtained using all of the observations available. As a result, the WLS algorithm is unsuitable for recursive tracking of time-varying frequencies, and its complexity grows with the number of measurements. To solve these problems, a new frequency estimator based on the Kalman filter is proposed.

At each time instant $n$, we consider a data segment consisting of $L$ past samples, $\overline{\mathbf{y}}_{n}=[y(n-L+1), \ldots, y(n)]$. If we assume that the frequencies of the sinusoidal signal does not change within the data segment $\overline{\mathbf{y}}_{n}$, then $\mathbf{Z}(n)$ and $\mathbf{Y}(n)$ can be derived from $\overline{\mathbf{y}}_{n}$ and the LP approach can be used to estimate the timedependent LPCs $\mathbf{b}(n)$ from

$$
\mathbf{Z}(n)=\mathbf{Y}(n) \mathbf{b}(n)+\mathbf{e}(n) .
$$

More precisely, let us rewrite $\overline{\mathbf{y}}_{\mathbf{n}}=[y(n-L+1), \ldots, y(n)]=$ $\left[y_{n}(1), \ldots, y_{n}(L)\right]$. Then, one obtains the equation shown at the botam) of theyp(ge- 1$)+y_{n}(L-2 M-1), \ldots, y_{n}(2 M)+$ $\left.y_{n}(0)\right]^{T}, \mathbf{b}(n)=\left[b_{1}(n), \ldots, b_{M}(n)\right]^{T}$, and the residual error $\mathbf{e}(n)=\left[e_{n}(L-1), e_{n}(L-2), \ldots, e_{n}(2 M)\right]^{T}$ with entries

$$
\begin{aligned}
e_{n}(\tau)= & y_{n}(\tau)+y_{n}(\tau-2 M)-\sum_{i=1}^{M-1} b_{i}(n) \\
& \times\left[y_{n}(\tau-i)+y_{n}(\tau-2 M+i)\right]-b_{M}(n) y_{n}(\tau-M) \\
= & -\sum_{i=0}^{M-1} b_{i}(n)[q(\tau-L+n-i)+q(\tau-L+n-2 M+i)] \\
& -b_{M}(n) q(\tau-L+n-M) .
\end{aligned}
$$

Let $\mathbf{R}_{e}(n)$ be the covariance matrix of the correlated residual $\mathbf{e}(n)$. The p.d.f. of $\mathbf{e}(n)$ can be written as

$$
p(\mathbf{e}(n))=\frac{1}{\left(2 \pi\left|\mathbf{R}_{e}(n)\right|\right)^{(L-2 M) / 2}} \exp \left(-\frac{1}{2} \mathbf{e}^{T}(n) \mathbf{R}_{e}^{-1}(n) \mathbf{e}(n)\right)
$$

which suggests the following LS estimate:

$$
\hat{\mathbf{b}}(n)=\arg \min _{\hat{\mathbf{b}}} \sum_{i=1}^{n} \lambda^{n-i} \cdot \mathbf{e}^{T}(i) \mathbf{R}_{e}^{-1}(i) \mathbf{e}(i)
$$

where $\lambda$ is a forgetting factor to forget samples in the distant past for tracking purposes.

A time-recursive frequency estimator based on the WRLS algorithm was proposed in [8] to estimate $\mathbf{b}(n)$ and the Cholesky factor of $\mathbf{R}_{e}(n)$. The conventional RLS algorithm can be regarded as the WRLS algorithm with $\mathbf{R}_{e}(n)=\mathbf{I}$. For the LP-based frequency estimator problem, the RLS method will be degraded due to the colored noise.

The Kalman filter can also be used to estimate $\mathbf{b}(n)$ of (12) recursively, if we assume that $\mathbf{b}(n)$ follows a simple one-order AR model with smoothness priors [14]. Consequently, the LPCs can be described as the state equation in (16a). Combining the state equation with the LP equation in (12) gives the following linear state-space model:

$$
\left\{\begin{array}{l}
\mathbf{b}(n)=\mathbf{F} \mathbf{b}(n-1)+\boldsymbol{\delta}(n) \\
\mathbf{Z}(n)=\mathbf{Y}(n) \mathbf{b}(n)+\mathbf{e}(n)
\end{array}\right.
$$

where $\mathbf{b}(n)$ is the system state. For simplicity, the state transition matrix $\mathbf{F}$ is chosen as an identity matrix and the variation of the LPCs is modeled by the state noise vector $\boldsymbol{\delta}(n)$, which is assumed to be zero mean Gaussian distributed random vector with covariance matrix $\mathbf{R}_{\delta}(n)$. The correlated residual $\mathbf{e}(n)$ is the measurement noise with covariance $\mathbf{R}_{e}(n)$.

The optimal state estimator for $\hat{\mathbf{b}}(n)$ in the MMSE criterion for the state-space model (16) can be computed by the standard Kalman filter recursions as

$$
\begin{aligned}
\mathbf{e}(n)= & \mathbf{Z}(n)-\mathbf{Y}(n) \hat{\mathbf{b}}(n /(n-1)) \\
\mathbf{K}(n)= & \mathbf{P}(n /(n-1)) \mathbf{Y}^{T}(n) \\
& \cdot\left[\mathbf{R}_{e}(n)+\mathbf{Y}(n) \mathbf{P}(n /(n-1)) \mathbf{Y}^{T}(n)\right]^{-1} \\
\hat{\mathbf{b}}(n / n)= & \hat{\mathbf{b}}(n /(n-1))+\mathbf{K}(n) \mathbf{e}(n) \\
\mathbf{P}(n / n)= & {[\mathbf{I}-\mathbf{K}(n) \mathbf{Y}(n)] \cdot \mathbf{P}(n /(n-1)) } \\
\hat{\mathbf{b}}((n+1) / n)= & \mathbf{F} \mathbf{b}(n / n) \\
\mathbf{P}((n+1) / n)= & \mathbf{F P}(n / n) \mathbf{F}^{T}+\mathbf{R}_{\delta}(n)
\end{aligned}
$$

where $\hat{\mathbf{b}}(n)=\hat{\mathbf{b}}(n / n), \hat{\mathbf{b}}(n / \tau)(\tau=n-1$ or $n)$ represents the estimator of $\mathbf{b}(n)$ given the measurements up to time $\tau, \mathbf{P}(n / \tau)$ is the autocorrelation matrix of $\hat{\mathbf{b}}(n / \tau)$, and $\mathbf{K}(n)$ is the Kalman gain. To ensure at least one measurement is included, the data length of $\overline{\mathbf{y}}_{n}$ should satisfy $L>2 M$.

\section{NoISE COVARIANCE ESTIMATION AND AN ITERATIVE FREQUENCY ESTIMATOR}

In the proposed Kalman-filter-based frequency estimator above, the covariance matrices of the measurement and state

$$
\mathbf{Y}(n)=\left[\begin{array}{ccccc}
y_{n}(L-2)+y_{n}(L-2 M) & y_{n}(L-3)+y_{n}(L-2 M+1) & & y_{n}(L-M)+y_{n}(L-M-2) & y_{n}(L-M-1) \\
\vdots & \vdots & \ddots & \vdots & \vdots \\
y_{n}(2 M-1)+y_{n}(1) & y_{n}(2 M-2)+y_{n}(2) & & y_{n}(M+1)+y_{n}(M-1) & y_{n}(M)
\end{array}\right]
$$


noise, $\mathbf{R}_{e}(n)$ and $\mathbf{R}_{\delta}(n)$, play an important role. Since they are not known a priori, they should be estimated. Here, we will discuss the recursive estimation of the noise covariance matrices and develop a Kalman-filter-based iterative frequency estimator to improve the estimation accuracy.

First of all, we consider the covariance matrix $\mathbf{R}_{e}(n)$ of the measurement noise $\mathbf{e}(n)$, which will be estimated from $\mathbf{e}(n)=$ $\mathbf{Z}(n)-\mathbf{Y}(n) \hat{b}(n)$. If we assume that $\mathbf{e}(n)$ is ergodic, then its ensemble average can be replaced by time average. Hence, the covariance of $\mathbf{e}(n)$ can be estimated recursively $\operatorname{as}_{e}(n)=$ $\lambda_{e} \mathbf{R}_{e}(n-1)+\left(1-\lambda_{e}\right) \mathbf{e}(n) \mathbf{e}^{H}(n)$, where a forgetting factor $\lambda_{e}$ is introduced in the recursive update. If all of the errors in a window of length $N_{e}$ are used to reduce the variance in estimating the covariance matrix, the calculation of $\mathbf{R}_{e}(n)$ can further be modified to

$$
\mathbf{R}_{e}(n)=\lambda_{e} \mathbf{R}_{e}(n-1)+\left(1-\lambda_{e}\right) \widetilde{\mathbf{R}}_{e}(n)
$$

where $\widetilde{\mathbf{R}}_{e}(n)$ is the covariance matrix of the residual error matrix $\left[\mathbf{e}\left(n-N_{e}+1\right), \ldots, \mathbf{e}(n-1), \mathbf{e}(n)\right]$.

A similar approach based on (16a) suggests the state noise estimate $\boldsymbol{\delta}(n)=\hat{\mathbf{b}}(n)-\mathbf{F} \hat{\mathbf{b}}(n-1)$. Therefore, the state noise covariance matrix $\mathbf{R}_{\delta}(n)=E\left[\boldsymbol{\delta}(n) \boldsymbol{\delta}^{H}(n)\right]$ can be estimated recursively from

$$
\mathbf{R}_{\delta}(n)=\lambda_{\delta} \mathbf{R}_{\delta}(n-1)+\left(1-\lambda_{\delta}\right) \widetilde{\mathbf{R}}_{\delta}(n)
$$

where $\lambda_{\delta}$ is the forgetting factor, and $\widetilde{\mathbf{R}}_{\delta}(n)$ is the covariance matrix of $\left[\boldsymbol{\delta}\left(n-N_{\delta}+1\right), \ldots, \boldsymbol{\delta}(n-1), \boldsymbol{\delta}(n)\right]$ with an estimation window of length $N_{\delta}$.

Note that the forgetting factors $\lambda_{e}$ and $\lambda_{\delta}$ should be slightly less than one so that the effects of previous estimations/observations can be gradually neglected. However, the optimal forgetting factor selection is beyond our scope, and thus we set all of these forgetting factors to 0.95 in the simulations.

In [8], $\mathbf{b}(n)$ and $\mathbf{R}_{e}(n)$ were estimated iteratively in the WRLS-based frequency estimator so that a better result can be achieved when the linear prediction error $\mathbf{e}(n)$ is colored. A similar iterative algorithm can be applied to the above Kalman filter-based algorithm to improve the estimation accuracy of $\mathbf{R}_{e}(n)$ and hence $\mathbf{b}(n)$.

The detailed procedure of this Kalman-filter-based iterative algorithm is summarized in Table I. Compared with the WRLSbased iterative algorithm of [8], the Kalman-filter-based iterative algorithm is more convenient because $\mathbf{R}_{e}(n)$ can be directly incorporated in the Kalman gain matrix. Simulation results show that the Kalman-filter-based iterative algorithm usually converges in three to five iterations and it can improve considerably the tracking accuracy.

At each time instant, the major arithmetic complexity of the proposed algorithm comes from the matrix inversion, which requires a complexity of $O\left((L-2 M)^{3}\right)$, for computing the Kalman gain in (17b). Hence, the total complexity is approximately $O\left(k_{\max } N(L-2 M)^{3}\right)$, which is comparable to the WRLS iterative algorithm in [8]. For the WLS algorithm in [7], its order of complexity is up to $O\left(N^{3}\right)$, and therefore the complexity grows significantly with the total number of measurements.
TABLE I

KALMAN-FILTER-BASED ITERATIVE FREQUENCY ESTIMATOR

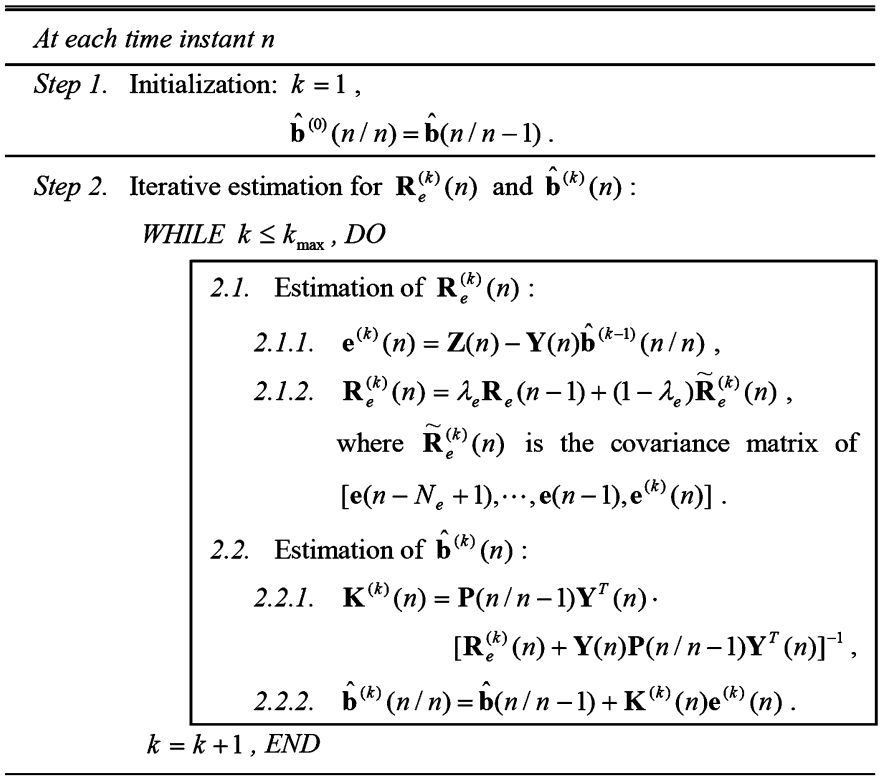

Step 3. Estimation of $\mathbf{R}_{\delta}(n)$ : same as (19).

Step 4. Update of $\hat{\mathbf{b}}(n+1 / n)$ and $\mathbf{P}(n+1 / n)$ : same as (17d, e, and f).

\section{Simulation Results}

The performance of the proposed Kalman-filter-based frequency estimator is evaluated using computer simulations. Four different frequency estimators are tested for different sinusoidal signals: 1) RLS-based estimator; 2) WRLS-based iterative estimator $\left(k_{\max }=5\right)$; 3 ) the Kalman-filter-based estimators without iterations $\left(k_{\max }=1\right)$; and 4) the Kalman-filter-based estimators with iterations $\left(k_{\max }=5\right)$, .

The length of the input signal is $N=1000$ and the sampling rate is 1 . The sinusoidal signals are assumed to consist of two components $(M=2)$ and their amplitudes and phases are $\alpha_{m}=[1.5,1]$ and $\phi_{m}=[0, \pi / 3]$, respectively. The forgetting factor $\lambda$ of the RLS- and WRLS-based algorithms is chosen as 0.95 . The length of data segment $\overline{\mathbf{y}}_{n}$ is set as $L=2 M+1=5$ to reduce the arithmetic complexity. The parameters for the noise covariance matrix estimation (18) and (19) are chosen as $\lambda_{e}=\lambda_{\delta}=0.95$ and $N_{e}=N_{\delta}=L$.

The MSE criterion is used to evaluate the performances of the estimation, and it is given by $\operatorname{MSE}(n)=$ $10 \log _{10}\left(\left\|w_{m}(n)-\hat{w}_{m}(n)\right\|_{2}^{2}\right)$, where $\hat{w}_{m}(n)$ is the estimated frequencies. The MSE results shown in the figures are averages of 1000 independent Monte Carlo runs.

The first experiment uses stationary sinusoids with fixed frequencies to demonstrate the performance of these frequency estimators. The two frequencies $w_{m}$ are chosen randomly from $(0,0.5)$. The test signals are corrupted by a zero-mean AWGN with a SNR of $10 \mathrm{~dB}$. In Fig. 1(a), the Kalman-filter-based frequency estimators achieve better tracking results than the RLSand WRLS-based estimators. It can also be seen that more iterations in the Kalman-filter-based algorithm will improve the tracking accuracy and the convergence rate. 


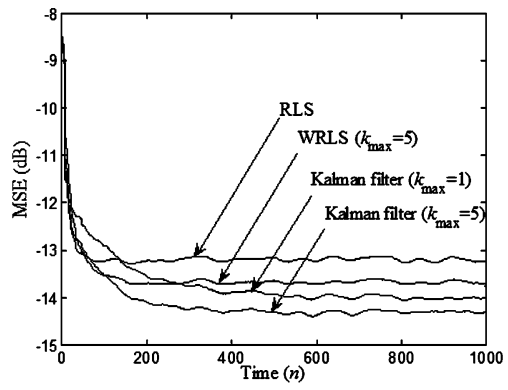

(a)

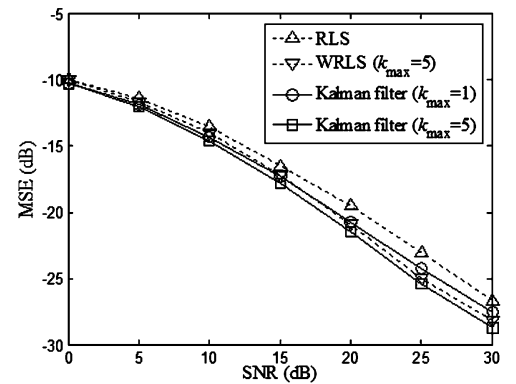

(b)

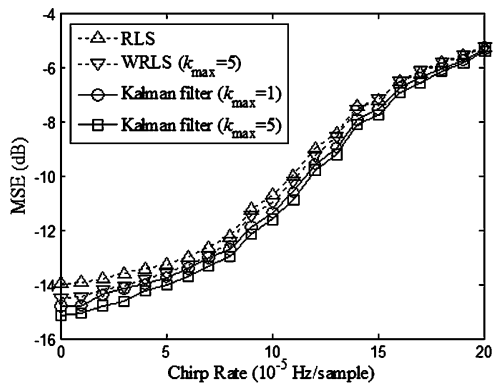

(c)

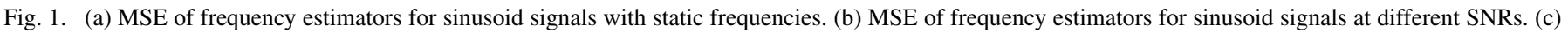
MSE of frequency estimators for signals with different chirp rates.

Next, we compare the performances of these frequency estimators for stationary sinusoids whose frequencies are also randomly chosen in the range $(0,0.5)$ and are contaminated with AWGNs of different SNRs. Fig. 1(b) shows the steady-state MSE results of various algorithms at time instant 1000. We can see that the Kalman-filter-based algorithm with five iterations provides the best performance, while that of the RLS-based method is the worst. It is also noted that the performance of the iterative WRLS estimator is slightly better than the Kalmanfilter-based estimator without iteration when SNR is sufficiently high $(\geq 20 \mathrm{~dB})$ due to its long effective window length. Therefore, more iteration processes for estimating the noise covariance are particularly useful when the additive noise is small.

\section{CONCLUSION}

A Kalman-filter-based iterative algorithm for tracking timevarying frequencies of sinusoidal signals was presented. This algorithm was based on the linear prediction approach and the LPCs were estimated using the Kalman filter recursively. Moreover, an iterative algorithm for refining the LPCs and the measurement noise covariance matrix was employed to improve the tracking performance. Simulation results showed that the proposed Kalman-filter-based frequency estimator had a better performance than the RLS- and WRLS-based algorithms for signals at different SNRs and frequency variations.

\section{REFERENCES}

[1] A. Watanabe, "Formant estimation method using inverse-filter control," IEEE Trans. Speech Audio Process., vol. 9, no. 4, pp. 317-326, Nov. 2001.

[2] J. F. Thayer, J. J. Sollers, III, E. R. Padial, and J. Vila, "Estimating respiratory frequency from autoregressive spectral analysis of heart period," IEEE Eng. Med. Biol. Mag., vol. 21, no. 4, pp. 41-45, Apr. 2002.

[3] J. Proakis et al., Algorithms for Statistical Signal Processing. Englewood Cliffs, NJ: Prentice-Hall, 2002.

[4] L. L. Scharf, Statistical Signal Processing: Detection, Estimation, and Time Series Analysis. Reading, MA: Addison-Wesley, 1991.

[5] X. Xia, "Global frequency estimation using adaptive identifiers," IEEE Trans. Automat. Contr., vol. 47, no. 7, pp. 1188-1193, Jul. 2002.

[6] A. Bobtsov, "New approach to the problem of globally convergent frequency estimator," Int. J. Adapt. Control Signal Process., 2007.

[7] H. C. So, K. W. Chan, Y. T. Chan, and K. C. Ho, "Linear prediction approach for efficient frequency estimation of multiple real sinusoids: Algorithms and analyses," IEEE Trans. Signal Process., vol. 53, no. 7, pp. 2290-2305, Jul. 2005.

[8] W. Y. Lau, S. C. Chan, Z. G. Zhang, and C. H. Leung, "A new QR-decomposition based recursive frequency estimator for multiple sinusoids in impulsive noise environment," in Proc. IEEE Int. Symp. Circuits Syst., Kos, Greece, May 21-24, 2006, pp. 4248-4251.

[9] T. Söderstrom and P. Stoica, System Identification. Englewood Cliffs, NJ: Prentice-Hall, 1989.

[10] G. C. Goodwin and R. L. Payne, Dynamic System Identification: Experiment Design and Data Analysis. New York: Academic, 1977.

[11] S. M. Kay, Fundamentals of Statistical Signal Processing: Estimation Theory. Upper Saddle River, NJ: Prentice-Hall, 1993.

[12] D. Lippuner and G. S. Moschytz, "The Kalman filter in the context of adaptive filter theory," Int. J. Circuit Theory Appl., vol. 32, no. 4, pp. 223-253, 2004.

[13] Y. T. Chan, J. M. M. Lavoie, and J. B. Plant, "A parameter estimation approach to estimation of frequencies of sinusoids," IEEE Trans. Acoust. Speech, Signal Process., vol. 29, no. 2, pp. 214-219, Apr. 1981.

[14] G. Kitagawa and W. Gersch, "A smoothness priors time-varying AR coefficient modeling of nonstationary covariance time series," IEEE Trans. Automat. Contr., vol. AC-30, no. 1, pp. 48-56, Jan. 1985. 